\title{
Cognitive outcomes after unruptured intracranial aneurysm treatment with flow diversion
}

\author{
Kathryn Wagner, MD, ${ }^{1}$ Aditya Srivatsan, MS, ${ }^{1}$ Alina Mohanty, BA, ${ }^{1}$ Visish M. Srinivasan, MD, ${ }^{1}$ \\ Yasir Saleem, MD, ${ }^{2}$ Jacob Cherian, MD, ${ }^{4}$ Robert F. James, MD, ${ }^{5}$ Stephen Chen, MD, ${ }^{3}$ \\ Jan-Karl Burkhardt, MD, ${ }^{1}$ Jeremiah Johnson, MD, ${ }^{1}$ and Peter Kan, MD${ }^{1}$
}

Departments of ${ }^{1}$ Neurosurgery, ${ }^{2}$ Neurology, and ${ }^{3}$ Radiology, Baylor College of Medicine, Houston, Texas; ${ }^{4}$ Department of Neurosurgery, Emory University, Atlanta, Georgia; and ${ }^{5}$ Department of Neurosurgery, University of Louisville, Kentucky

OBJECTIVE Flow diversion is increasingly used to treat a variety of intracranial aneurysms with good safety and efficacy; however, there is some evidence that this treatment is associated with a larger postoperative ischemic burden on imaging than that with other traditional endovascular modalities. These findings typically do not manifest as neurological deficits, but any subtle effects on cognition remain unknown. In this study, the authors describe the neurocognitive performance of a cohort of patients with unruptured intracranial aneurysms (UIAs) before and after treatment with flow diversion. This is the first report of cognitive outcomes following aneurysm treatment with flow diversion.

METHODS The authors prospectively collected data on cognitive function using the Montreal Cognitive Assessment (MoCA) tool in patients with UIAs who were undergoing endovascular aneurysm treatment with flow diversion between June 2017 and July 2019. Patients completed the MoCA prior to intervention, at the 1-month follow-up after treatment, and again at 6 months after the procedure. All patients with UIAs treated with flow diversion were included regardless of age, aneurysm location, or morphology, unless their functional status precluded completion of the MoCA instrument. A repeated-measures linear mixed-effects model was used to compare preintervention and postintervention cognitive status at the time intervals outlined.

RESULTS Fifty-one patients with 61 aneurysms underwent endovascular aneurysm treatment with flow diversion (mean age 52.5 years, $90.2 \%$ females). There was no difference between baseline and postprocedure MoCA scores at any time interval $(p>0.05)$. The MoCA scores at baseline, 1 month postprocedure, and 6 months postprocedure were 26.1, 26.2 , and 26.6, respectively. There was also no difference between pre- and postprocedure scores on any individual domain of the instrument (visuospatial, naming, attention, language, abstraction, delayed recall, and orientation) at any time interval $(p>0.05)$. Thirty-four patients had follow-up MRI or CT imaging, 5 of whom showed radiographic changes or ischemia. All patients with follow-up clinical evaluation had a 6-month modified Rankin Scale score $\leq 2$.

CONCLUSIONS Flow diversion is increasingly used in the treatment of intracranial aneurysms. This study suggests that this treatment may not alter neurocognitive function. Larger patient samples and longer follow-ups with other tests of cognitive functions are needed to confirm these findings.

https://thejns.org/doi/abs/10.3171/2019.9.JNS191910

KEYWORDS unruptured intracranial aneurysm; flow diversion; Pipeline embolization device; cognition; MoCA; Montreal Cognitive Assessment; vascular disorders

$\mathrm{T}$ REATMENT options for unruptured intracranial aneurysms (UIAs) have evolved and improved over the past decades. Flow diversion (FD) is becoming increasingly favored for sidewall intracranial aneurysms. ${ }^{19-21}$ Initially approved for proximal large carotid artery aneurysms, the Pipeline embolization device (PED) has expanded indications including for smaller aneurysms up to the carotid terminus. ${ }^{15}$ As the safety and efficacy data accumulate, FD is increasingly used to treat intracranial aneurysms of various morphologies and locations.

Despite the procedure's high occlusion and low complication rates, there is evidence that FD results in radio-

ABBREVIATIONS FD = flow diversion; MoCA = Montreal Cognitive Assessment; $m R S=$ modified Rankin Scale; PED = Pipeline embolization device; RROC = RaymondRoy Occlusion Classification; UIA = unruptured intracranial aneurysm.

SUBMITTED July 11, 2019. ACCEPTED September 16, 2019.

INCLUDE WHEN CITING Published online November 29, 2019; DOI: 10.3171/2019.9.JNS191910. 
graphic findings of ischemia, evident on diffusion-weighted MRI. Established endovascular techniques, including stents and coils, have demonstrated silent infarct rates from $10 \%$ to $69 \%, 1,2,14,17$ but there is concern that these changes may be higher in patients treated with FD..$^{5,17,25,28}$ These patients generally do not experience correlative neurological symptoms; however, the long-term significance of these changes remains unknown. It has been posited that while there may not be motor, sensory, or speech deficits from these infarcts, there may be subtle neurocognitive effects that are less apparent. ${ }^{3,4,17,22,33}$

There has been no structured investigation into the cognitive outcomes of treatment with FD. Specifically, no data exist to determine if there is a measurable cognitive decline after treatment with FD or whether the decline, if one does exist, correlates with the reported radiographic abnormalities after the procedure.

Several validated tools are used to assess cognitive function. The Montreal Cognitive Assessment (MoCA) tool is a 30-point test that can be administered over approximately 15 minutes and is sensitive enough to detect mild cognitive deficits. ${ }^{8}$ There has been extensive validation of the sensitivity of this instrument in detecting mild cognitive impairment across a range of neurological pathologies, languages, and cultures. ${ }^{9,11,24,26} \mathrm{Here}$, we present the first study on patients' neurocognitive performance before and after unruptured aneurysm treatment with FD at different time intervals using the MoCA.

\section{Methods}

We prospectively collected data on cognitive function using the MoCA tool in all patients with UIAs who were undergoing endovascular treatment with FD in the period from June 2017 to July 2019. The study was approved by the institutional review boards and ethics committees at the participating institutions of Baylor College of Medicine, Emory University, and University of Louisville. Senior neurointerventional faculty evaluated clinical presentation and imaging studies and formulated the treatment plan for all patients.

All assessments were administered by trained neurosurgery team members independent of the study who had completed an online MoCA training course. We administered English version 1 as the patients' first and third MoCAs and English version 2 as their second in order to prevent a practice effect. Scores were recorded for each of the 7 subsections (visuospatial, naming, attention, language, abstraction, delayed recall, and orientation), as well as the total score. Patients completed the MoCA prior to intervention and at 1 and 6 months after the procedure at their regularly scheduled appointments. All patients with unruptured aneurysms treated with FD were included regardless of age, aneurysm location, or morphology, unless their functional status precluded completion of the MoCA instrument. Scores were compiled and analyzed using Stata version 15.1 (StataCorp LLC). A repeated-measures linear mixed-effects model was used to compare preintervention and postintervention cognitive status at the time intervals outlined. This model analyzes repeated measurements made on the same statistical unit and is similar to
TABLE 1. Demographic characteristics among 51 patients with UIAs treated with FD

\begin{tabular}{lc}
\hline \multicolumn{1}{c}{ Variable } & Value \\
\hline Age in yrs & \\
Mean \pm SD & $52.5 \pm 14.1$ \\
Range & $13-75$ \\
\hline Female sex, no. (\%) & $46(90.2)$ \\
\hline Race, no. (\%) & $30(58.8)$ \\
White & $12(23.5)$ \\
Hispanic & $3(5.9)$ \\
Asian & $6(11.8)$ \\
African American & \\
Clinical presentation, no. (\%) & $42(82.4)$ \\
Incidental & $2(3.9)$ \\
Headache & $3(5.9)$ \\
Aneurysm history & $3(5.9)$ \\
Diplopia & $1(1.9)$ \\
Facial tingling & \\
Smoker, no. (\%) & $31(60.8)$ \\
Never & $11(21.6)$ \\
Current & $9(17.6)$ \\
Former & \\
Family history, no. (\%) & $40(78.4)$ \\
No & $11(21.6)$ \\
Yes &
\end{tabular}

a repeated-measures ANOVA, although it is more robust in that both fixed and random effects are considered and missing data are better dealt with. A power analysis was also completed. A p value $<0.05$ was considered statistically significant.

The protocol for endovascular treatment with FD was published previously. ${ }^{31}$ Patients stayed 1 night in a neurosurgical intensive care unit and were discharged the following day if there were no complications. They were maintained on dual antiplatelet therapy for a minimum of 6 months following the procedure. Follow-up imaging was not obtained as part of a standard protocol but was performed at the discretion of the treating physician.

\section{Results}

A total of 51 patients with 61 aneurysms underwent endovascular aneurysm treatment with FD in the study period. There were 46 females and 5 males, with a mean age of 52.5 \pm 14.1 years and an age range of 13-75 years (Table 1). Presentations were largely incidental (42 patients [82.4\%]). Aneurysms were generally saccular (46 [90.2\%]; Table 2), ranging from 2 to $26 \mathrm{~mm}$ in size. Aneurysms varied widely in location, but ophthalmic aneurysms (24 patients [47.1\%]), posterior communicating artery aneurysms (7 [13.7\%]), and superior hypophyseal artery aneurysms (6 [11.8\%]) were the most common. Seven patients (13.7\%) had multiple aneurysms simultaneously treated with PEDs. Six patients (11.8\%) had two PEDs implanted, with the rest receiving a single device for treatment.

The mean MoCA score at baseline was $26.1 \pm 2.6$; at 
TABLE 2. Aneurysm characteristics

\begin{tabular}{cc}
\hline \multicolumn{1}{c}{ Variable } & No. (\%) \\
\hline Aneurysm type & $46(90.2)$ \\
\hline Saccular & $3(5.9)$ \\
\hline Fusiform & $2(3.9)$ \\
\hline Dissection related & $24(47.1)$ \\
\hline Aneurysm location & $7(13.7)$ \\
\hline Ophthalmic & $6(11.8)$ \\
\hline PCoA & $4(7.8)$ \\
\hline Superior hypophyseal & $2(3.9)$ \\
\hline Supraclinoid & $2(3.9)$ \\
\hline Cavernous & $2(3.9)$ \\
\hline M & $1(2.0)$ \\
\hline Carotid cave & $1(2.0)$ \\
\hline Paraclinoid & $1(2.0)$ \\
\hline ACoA & $1(2.0)$ \\
\hline Petrous & \\
\hline Intracranial vertebral & $7(13.7)$ \\
\hline No. of aneurysms treated w/ PED & $44(86.3)$ \\
\hline Multiple & $45(88.2)$ \\
\hline Single & $6(11.8)$ \\
\hline No. of PEDs used in procedure &
\end{tabular}

$\mathrm{ACoA}=$ anterior communicating artery; $\mathrm{PCoA}=$ posterior communicating artery. Values represent number (\%) of patients; there were 61 aneurysms among 51 patients.

1 month postprocedure, $26.2 \pm 2.5$; and at 6 months, 26.6 \pm 2.8 . All subcategory scores (visuospatial, naming, attention, language, abstraction, delayed recall, and orientation) were reported in Table 3 . There was no difference between baseline and postprocedure total MoCA scores at any time interval $(\mathrm{p}>0.05)$. There was also no difference between pre- and postprocedure scores on any individual domain of the instrument at any time point $(\mathrm{p}>0.05)$. Forty-four patients $(86.3 \%)$ completed a 1-month MoCA evaluation, and $26(51.0 \%)$ completed a 6-month MoCA. Only 4 patients completed a 1-year MoCA evaluation. The mean score for these patients prior to treatment was 26.5, and the 1-year posttreatment score was 26.8 .

Thirty-four patients $(66.7 \%)$ underwent postprocedure imaging, including CT/CTA $(n=19)$ and MRI $(n=15)$. Five of these patients (14.7\% of those imaged) showed new small areas of susceptibility on FLAIR sequences or diffusion restriction on MRI suggesting small areas of ischemia. One patient suffered an expected right frontal stroke after the pusher wire separated from the distal end and the distal end could not be retrieved. The mean MoCA scores of these 5 patients at 1 month and 6 months after treatment were 26.6 and 27.5 , respectively, which were not different from the scores in patients without radiographic changes (26.1 at both time points; $\mathrm{p}>0.05$ ).

Fifty patients remained clinically stable after the procedure, without changes in the motor or sensory examination. The aforementioned patient with a right frontal stroke initially suffered from left hemiparesis but only had mild hand weakness at the last follow-up. All patients with 6and 12-month clinical follow-ups had a modified Rankin Scale (mRS) score $\leq 2$. On the 6-month follow-up angiogram, aneurysm occlusion (measured using the RaymondRoy Occlusion Classification [RROC]) was class I in 18 patients, class II in 1 patient, and class III in 5 patients (Table 4). At the 12-month follow-up, 5 patients had class I occlusion and 1 patient had class III occlusion.

Aside from the pusher wire separation mentioned above, complications consisted of one retroperitoneal hematoma, which required no additional surgery or transfusion, and one delayed presentation of an atraumatic, asymptomatic intracerebral hemorrhage $(<1 \mathrm{~cm}) 1$ month after treatment, which was managed conservatively with observation (antiplatelet therapy continued). There was one death, a patient who had undergone uncomplicated treatment of a left ophthalmic segment aneurysm with PED. One year later, she presented in an obtunded state after rupture of her yet-untreated anterior communicating artery aneurysm, and her family elected to withdraw care. The ophthalmic aneurysm was occluded on CTA.

We also compared the latest aneurysm occlusion status to the latest MoCA score to see whether there was a correlation (Table 5). Patients with RROC class I had a mean MoCA score of 25.9 (range 18-30, SD 2.8), and those with RROC class III had a mean MoCA score of 26.5 (range 24-28, SD 1.4). No patient had a final RROC class II. There was no correlation between MoCA score and degree of aneurysm occlusion.

A power analysis was conducted to deduce the sample size required to detect a clinically relevant difference in patient MoCA scores pre- and post-PED treatment. A decrease in the MoCA score $\geq 2$ is considered clinically relevant. ${ }^{8,11}$ For an alpha value of 0.05 and a beta value of 0.8 , 27 patients are required in each group ( $\geq 27$ baseline patients and $\geq 27$ postoperative follow-up patients). Thus, our study was adequately powered to detect a difference of 2

TABLE 3. Average MoCA scores at each time point

\begin{tabular}{|c|c|c|c|c|c|c|c|c|}
\hline \multirow{2}{*}{$\begin{array}{c}\text { Mean MoCA } \\
\text { Score }\end{array}$} & \multicolumn{8}{|c|}{ Category (max points per category) } \\
\hline & VSE (5) & $\mathrm{N}(3)$ & Att (6) & $L(3)$ & Abs (2) & $\mathrm{DR}(5)$ & $O(6)$ & Score $(30)$ \\
\hline Preop & $4.4 \pm 1.0$ & $3.0 \pm 0.1$ & $5.3 \pm 0.9$ & $2.7 \pm 0.6$ & $1.8 \pm 0.5$ & $2.8 \pm 1.6$ & $5.9 \pm 0.2$ & $26.1 \pm 2.6$ \\
\hline 1-mo postop & $4.6 \pm 0.7$ & 3.0 & $5.2 \pm 1.2$ & $2.7 \pm 0.7$ & $2.0 \pm 0.2$ & $2.9 \pm 1.6$ & $5.9 \pm 0.3$ & $26.2 \pm 2.5$ \\
\hline 6-mo postop & $4.3 \pm 0.8$ & 3.0 & $5.2 \pm 1.1$ & $2.8 \pm 0.4$ & $2.0 \pm 0.2$ & $3.5 \pm 1.4$ & $5.8 \pm 0.4$ & $26.6 \pm 2.8$ \\
\hline
\end{tabular}

$\mathrm{Abs}=$ abstraction; Att = attention; $\mathrm{DR}=$ delayed recall $\mathrm{L}=$ = language $\mathrm{N}=$ naming; $\mathrm{O}=$ orientation $; \mathrm{VSE}=$ visuospatial . 
TABLE 4. Clinical, angiographic, and radiographic follow-up

\begin{tabular}{|c|c|}
\hline Variable & Value \\
\hline \multicolumn{2}{|l|}{ Preop mRS score } \\
\hline$\leq 2$ & $100 \%$ \\
\hline$>2$ & $0 \%$ \\
\hline \multicolumn{2}{|l|}{ Postop mRS score } \\
\hline$\leq 2$ & $98.0 \%$ \\
\hline$>2$ & $2.0 \%$ \\
\hline \multicolumn{2}{|l|}{ 6-mo postop mRS score } \\
\hline$\leq 2$ & $100 \%$ \\
\hline$>2$ & $0 \%$ \\
\hline \multicolumn{2}{|l|}{ 12-mo postop mRS score } \\
\hline$\leq 2$ & $100 \%$ \\
\hline$>2$ & $0 \%$ \\
\hline \multicolumn{2}{|l|}{ 6-mo postop RROC class } \\
\hline I & $18(75 \%)$ \\
\hline II & $1(4.2 \%)$ \\
\hline III & $5(20.8 \%)$ \\
\hline \multicolumn{2}{|l|}{ 12-mo postop RROC class } \\
\hline I & $5(83.3 \%)$ \\
\hline II & $0(0 \%)$ \\
\hline III & $1(16.7 \%)$ \\
\hline FU imaging & 34 \\
\hline MRI & $15(44.1 \%)$ \\
\hline CT/CTA & $19(55.9 \%)$ \\
\hline Radiographic changes & $5(14.7 \%)$ \\
\hline
\end{tabular}

$\mathrm{FU}=$ follow-up.

points in the MoCA for the comparison between baseline and 1 month postintervention but not for the comparison between baseline and 6 months postintervention (although it was close, since 26 patients had a 6-month follow-up).

\section{Discussion}

Here, we present the first study on cognitive outcomes following aneurysm treatment with FD. This preliminary study suggests that there is no apparent cognitive decline in the first 6 months following UIA treatment with FD while using the MoCA as the cognitive assessment tool. There were no changes in the total score, nor any differences in any subsection of the assessment. Only 4 patients had the MoCA at 1 year, with no apparent difference over that time; however, this number is not sufficient for statistical analysis. In the patients in whom follow-up imaging was performed, $14.7 \%$ showed radiographic changes of acute ischemia, but only 1 patient was symptomatic from a procedural complication leading to a frontal stroke. The remaining patients maintained their neurological and neurocognitive baseline levels, regardless of their imaging findings. Our complication and aneurysm occlusion rates are similar to those established in the literature..$^{13,21,23} \mathrm{In}$ the subset of patients who had 6- or 12-month follow-up angiograms (26 [51.0\%] of 51 patients), we found no correlation between latest RROC class and latest MoCA score.
TABLE 5. Latest RROC class versus the latest MoCA score

\begin{tabular}{cc}
\hline Latest RROC class & Latest MoCA Score \\
\hline I & $25.9 \pm 2.8$ \\
\hline III & $26.5 \pm 1.4$ \\
\hline
\end{tabular}

The MoCA tool has been assessed and validated to detect even mild changes in neurocognitive function. It is easy to administer and can even be used to evaluate patients with preexisting deficits or disabilities. It does not require formal training or a special environment. However, it is not designed for evaluation of higher cognitive functioning, executive skills, or personality. These are other dimensions that can be affected by small ischemic events and can seriously detract from quality of life. To fully evaluate these areas, patients would need to undergo extensive, formal neurocognitive and neuropsychiatric testing. The Hospital Anxiety and Depression Scale (HADS) and civilian Posttraumatic Stress Disorder (PTSD) Scale are other validated instruments that have been used to assess neurocognitive and neuropsychiatric outcomes after cerebral aneurysm treatment. ${ }^{30}$

Aneurysm treatment with any approach can lead to cognitive decline, although this depends on the treatment modality and patient presentation. The data on cognitive outcomes following treatment of UIAs are both small and conflicting. ${ }^{47}$ There is some evidence to suggest that the neurocognitive decline following endovascular therapies is less than that with open microsurgical treatment, ${ }^{7}$ but this is far from conclusive., ${ }^{4,10,27,29}$ There has even been some evidence that patients harboring intracranial aneurysms have lower baseline cognitive function. ${ }^{32}$ In fact, our cohort's mean baseline MoCA score of 26.1 nearly met the cutoff score of 26 for mild cognitive impairment, ${ }^{16}$ even though all of the patients had a baseline mRS score and NIH Stroke Scale (NIHSS) score of 0, emphasizing that simple clinical scales such as the mRS and NIHSS may be insufficient to assess global neurological function. It is unclear whether this is attributable to the lesion itself or whether less-quantitative aspects, such as stress or anxiety, play a role. Some studies have suggested that rates of depression and anxiety are higher in patients harboring UIAs ${ }^{29,30}$ but this has yet to be correlated with cognitive function. Nonetheless, the existence of the pathology in the absence of rupture or treatment complications may predispose patients to limitations in neurocognition.

The frequency of silent infarcts from aneurysm treatment with FD may be higher than that with other endovascular techniques. One study prospectively evaluated FLAIR and diffusion-weighted imaging (DWI) changes in 41 patients treated with FD and showed that those treated with FD were significantly more likely to have radiographic acute ischemic changes than those treated with standard stent-assisted coiling ( $54 \%$ vs $14 \%$, p < 0.001). However, these lesions were generally hemispheric and attributed to embolic phenomena, and patients did not experience correlative neurological symptoms. ${ }^{28} \mathrm{~A}$ second study found DWI changes in $92 \%$ of patients treated with FD; however, these were only clinically significant in $13 \%$, and most patients showed no clinical symptoms.${ }^{17}$ Brasiliense et al. 
evaluated 59 patients treated with FD, with MRI showing evidence of ischemia in $63 \%$; however, $21 \%$ of cases were not in the vascular territory of the vessel treated, and only $5 \%$ experienced neurological symptoms. ${ }^{6}$

In our cohort, $14.7 \%$ of patients who underwent posttreatment imaging showed ischemic changes on postoperative imaging. The majority of them were clinically silent, and only one patient had a clinical deficit from a right frontal stroke. Although not all patients completed postoperative imaging, the incidence of postoperative ischemic change on imaging in our cohort appears to be less than that reported in the literature. This can be explained by the fact that $55.9 \%$ of the postoperative imaging was $\mathrm{CT}$, which has limited sensitivity in detecting small silent ischemic changes. Nonetheless, our cohort showed no significant change in neurocognitive performance regardless of imaging findings, suggesting that the clinically silent ischemic burden often seen on postoperative MRI does not appear to negatively impact neurological function.

Decisions regarding the treatment of UIAs must weigh the risk of intervention against the natural history of the lesion and the risk of rupture. These risks are multifactorial and depend on a multitude of patient factors and aneurysm characteristics. ${ }^{12,18}$ Most UIAs carry a low risk of hemorrhage, ${ }^{1,17}$ and the treatment risk must therefore also be low. Thus, determining the UIA treatment effect on cognitive outcome is particularly important. If a clear effect of treatment on cognitive function exists, whether improvement or impairment, then this becomes a critical factor to be considered in the decision analysis of whether or not to treat UIAs with a particular modality. Our preliminary study suggests that there is no apparent cognitive decline in the first 6 months following UIA treatment with FD.

\section{Study Limitations}

Our study was limited by a small sample size, with short-term and incomplete follow-up for those who were studied. Additionally, only a single neurocognitive examination was administered, and there was no standard postoperative imaging. To address the study's weaknesses, future studies should include a larger patient sample and use a more extended battery of validated neuropsychological tests (MoCA, HADS, and PTSD Scale for example). For each patient, a longer follow-up (12 months) is preferable along with standardized postoperative MRI to document ischemic lesions.

\section{Conclusions}

Patients with UIAs who undergo FD do not appear to have detrimental effects on cognitive function after treatment. Larger patient samples and longer follow-ups with other tests of cognitive functions are needed to confirm these findings.

\section{References}

1. Altay T, Kang HI, Woo HH, Masaryk TJ, Rasmussen PA, Fiorella DJ, et al: Thromboembolic events associated with endovascular treatment of cerebral aneurysms. J Neurointerv Surg 3:147-150, 2011

2. Biondi A, Oppenheim C, Vivas E, Casasco A, Lalam T,
Sourour N, et al: Cerebral aneurysms treated by Guglielmi detachable coils: evaluation with diffusion-weighted MR imaging. AJNR Am J Neuroradiol 21:957-963, 2000

3. Bonares MJ, de Oliveira Manoel AL, Macdonald RL, Schweizer TA: Behavioral profile of unruptured intracranial aneurysms: a systematic review. Ann Clin Transl Neurol 1:220-232, 2014

4. Bonares MJ, Egeto P, de Oliveira Manoel AL, Vesely KA, Macdonald RL, Schweizer TA: Unruptured intracranial aneurysm treatment effects on cognitive function: a metaanalysis. J Neurosurg 124:784-790, 2016

5. Bond KM, Brinjikji W, Murad MH, Kallmes DF, Cloft HJ, Lanzino G: Diffusion-weighted imaging-detected ischemic lesions following endovascular treatment of cerebral aneurysms: a systematic review and meta-analysis. AJNR Am J Neuroradiol 38:304-309, 2017

6. Brasiliense LBC, Stanley MA, Grewal SS, Cloft HJ, Sauvageau $\mathrm{E}$, Lanzino $\mathrm{G}$, et al: Silent ischemic events after Pipeline embolization device: a prospective evaluation with MR diffusion-weighted imaging. J Neurointerv Surg 8:1136-1139, 2016

7. Bründl E, Böhm C, Lürding R, Schödel P, Bele S, Hochreiter $A$, et al: Treatment of unruptured intracranial aneurysms and cognitive performance: preliminary results of a prospective clinical trial. World Neurosurg 94:145-156, 2016

8. Ciesielska N, Sokołowski R, Mazur E, Podhorecka M, Polak-Szabela A, Kędziora-Kornatowska K: Is the Montreal Cognitive Assessment (MoCA) test better suited than the Mini-Mental State Examination (MMSE) in mild cognitive impairment (MCI) detection among people aged over 60? Meta-analysis. Psychiatr Pol 50:1039-1052, 2016

9. De Roeck EE, De Deyn PP, Dierckx E, Engelborghs S: Brief cognitive screening instruments for early detection of Alzheimer's disease: a systematic review. Alzheimers Res Ther 11:21, 2019

10. Fontanella M, Perozzo P, Ursone R, Garbossa D, Bergui M: Neuropsychological assessment after microsurgical clipping or endovascular treatment for anterior communicating artery aneurysm. Acta Neurochir (Wien) 145:867-872, 2003

11. Goldstein FC, Milloy A, Loring DW: Incremental validity of Montreal Cognitive Assessment index scores in mild cognitive impairment and Alzheimer disease. Dement Geriatr Cogn Disord 45:49-55, 2018

12. Greving JP, Wermer MJH, Brown RD Jr, Morita A, Juvela $\mathrm{S}$, Yonekura M, et al: Development of the PHASES score for prediction of risk of rupture of intracranial aneurysms: a pooled analysis of six prospective cohort studies. Lancet Neurol 13:59-66, 2014

13. Griessenauer CJ, Ogilvy CS, Foreman PM, Chua MH, Harrigan MR, He L, et al: Pipeline Embolization Device for small intracranial aneurysms: evaluation of safety and efficacy in a multicenter cohort. Neurosurgery 80:579-587, 2017

14. Hahnemann ML, Ringelstein A, Sandalcioglu IE, Goericke S, Moenninghoff C, Wanke I, et al: Silent embolism after stent-assisted coiling of cerebral aneurysms: diffusionweighted MRI study of 75 cases. J Neurointerv Surg 6:461-465, 2014

15. Hanel RA: Prospective, multi-center study of flow diversion for small and medium-sized aneurysms: results of the premier trial, presented at International Stroke Conference 2017, February 21-24, 2017 (https://professional.heart.org/ idc/groups/ahamah-public/@wcm/@sop/@scon/documents/ downloadable/ucm_492111.pdf) [Accessed October 7, 2019]

16. Hoops S, Nazem S, Siderowf AD, Duda JE, Xie SX, Stern MB, et al: Validity of the MoCA and MMSE in the detection of MCI and dementia in Parkinson disease. Neurology 73:1738-1745, 2009

17. Iosif C, Camilleri Y, Saleme S, Caire F, Yardin C, Ponomarjova $\mathrm{S}$, et al: Diffusion-weighted imaging-detected ischemic 
lesions associated with flow-diverting stents in intracranial aneurysms: safety, potential mechanisms, clinical outcome, and concerns. J Neurosurg 122:627-636, 2015

18. Juvela S, Porras M, Poussa K: Natural history of unruptured intracranial aneurysms: probability of and risk factors for aneurysm rupture. J Neurosurg 108:1052-1060, 2008

19. Kallmes DF, Brinjikji W, Boccardi E, Ciceri E, Diaz O, Tawk R, et al: Aneurysm Study of Pipeline in an Observational Registry (ASPIRe). Intervent Neurol 5:89-99, 2016

20. Kallmes DF, Brinjikji W, Cekirge S, Fiorella D, Hanel RA, Jabbour P, et al: Safety and efficacy of the Pipeline embolization device for treatment of intracranial aneurysms: a pooled analysis of 3 large studies. J Neurosurg 127:775-780, 2017

21. Kallmes DF, Hanel R, Lopes D, Boccardi E, Bonafé A, Cekirge S, et al: International retrospective study of the Pipeline Embolization Device: a multicenter aneurysm treatment study. AJNR Am J Neuroradiol 36:108-115, 2015

22. Kang DH, Hwang YH, Kim YS, Bae GY, Lee SJ: Cognitive outcome and clinically silent thromboembolic events after coiling of asymptomatic unruptured intracranial aneurysms. Neurosurgery 72:638-645, 2013

23. Lanzino G, Crobeddu E, Cloft HJ, Hanel R, Kallmes DF: Efficacy and safety of flow diversion for paraclinoid aneurysms: a matched-pair analysis compared with standard endovascular approaches. AJNR Am J Neuroradiol 33:2158-2161, 2012

24. McDicken JA, Elliott E, Blayney G, Makin S, Ali M, Larner AJ, et al: Accuracy of the short-form Montreal Cognitive Assessment: systematic review and validation. Int J Geriatr Psychiatry 34:1515-1525, 2019

25. McGuinness BJ, Memon S, Hope JK: Prospective study of early MRI appearances following flow-diverting stent placement for intracranial aneurysms. AJNR Am J Neuroradiol 36:943-948, 2015

26. Nasreddine ZS, Phillips NA, Bédirian V, Charbonneau S, Whitehead V, Collin I, et al: The Montreal Cognitive Assessment, MoCA: a brief screening tool for mild cognitive impairment. J Am Geriatr Soc 53:695-699, 2005

27. Pereira-Filho AA, Pereira AG, Pereira-Filho NA, Lima LC, Costa JCD, Kraemer JL, et al: Long-term behavioral and cognitive outcomes following clipping for incidental unruptured intracranial aneurysms. Neuropsychology 28:75-83, 2014

28. Safain MG, Roguski M, Heller RS, Malek AM: Flow diverter therapy with the Pipeline Embolization device is associated with an elevated rate of delayed fluid-attenuated inversion recovery lesions. Stroke 47:789-797, 2016
29. Towgood K, Ogden JA, Mee E: Psychosocial effects of harboring an untreated unruptured intracranial aneurysm. Neurosurgery 57:858-866, 2005

30. van der Schaaf IC, Brilstra EH, Rinkel GJE, Bossuyt PM, van Gijn J: Quality of life, anxiety, and depression in patients with an untreated intracranial aneurysm or arteriovenous malformation. Stroke 33:440-443, 2002

31. Wagner KM, Srinivasan VM, Srivatsan A, Ghali MGZ, Thomas AJ, Enriquez-Marulanda A, et al: Outcomes after coverage of lenticulostriate vessels by flow diverters: a multicenter experience. J Neurosurg [epub ahead of print January 11, 2019. DOI: $10.3171 / 2018.8$.JNS18755]

32. Zaki Ghali MG, Srinivasan VM, Wagner K, Rao C, Chen SR, Johnson JN, et al: Cognitive sequelae of unruptured and ruptured intracranial aneurysms and their treatment: modalities for neuropsychological assessment. World Neurosurg 120:537-549, 2018

\section{Disclosures}

This work was supported by a Joe Niekro Foundation grant. Dr. Kan is a consultant for Stryker and Cerenovus.

\section{Author Contributions}

Conception and design: Kan, Wagner, Chen, Johnson. Acquisition of data: Kan, Wagner, Srivatsan, Mohanty, Srinivasan, Cherian, James, Chen, Burkhardt, Johnson. Analysis and interpretation of data: Wagner, Srivatsan, Mohanty, Srinivasan, Saleem, Cherian, James, Chen. Drafting the article: Kan, Wagner, Srivatsan, Mohanty, Srinivasan, James, Johnson. Critically revising the article: all authors. Reviewed submitted version of manuscript: all authors. Approved the final version of the manuscript on behalf of all authors: Kan. Statistical analysis: Srivatsan. Administrative/ technical/material support: Kan. Study supervision: Kan.

\section{Correspondence}

Peter Kan: Baylor College of Medicine, Houston, TX. peter.kan@ bcm.edu. 